\title{
El síndrome de burnout en el personal docente de la Unidad Académica del Norte del Estado de Nayarit. Análisis comparativo 2018-2020
}

\section{The burnout syndrome in the teaching staff of the Academic Unit of the North of the State of Nayarit. Comparative analysis 2018-2020}

\author{
PARRA-GARCÍA, Rosa Ruth†*, CHÁVEZ-SÁNCHEZ, Haydee del Carmen y ZEA-VERDÍN, Aldo \\ Asunción
}

\author{
Universidad Autónoma de Nayarit, México. \\ ID $1^{\text {er }}$ Autor: Rosa Ruth, Parra-García \\ ID $1^{\mathrm{er}}$ Coautor: Haydee del Carmen, Chávez-Sánchez \\ ID $2^{\text {do }}$ Coautor: Aldo Asunción, Zea-Verdín
}

DOI: $10.35429 /$ JHS.2020.12.4.31.38

Recibido Julio 25, 2020; Aceptado Diciembre 30, 2020

\begin{abstract}
Resumen
El articulo presenta una investigación que se llevó a cabo con el personal docente de la Unidad Académica del Norte del Estado de Nayarit (UAEN) perteneciente a la Universidad Autónoma de Nayarit (UAN), con el objetivo de realizar un análisis comparativo sobre la incidencia del síndrome de Burnout en los años 2018 a 2020. El instrumento de recolección de información que se utilizó fue el cuestionario de Maslach Burnout Inventory el cual mide los siguientes aspectos: agotamiento emocional, despersonalización y realización personal. Como parte de los resultados se documentó un incremento del $10 \%$ de incidencia del Síndrome de Burnout en el personal docente, el cual se atribuye a las condiciones de trabajo provocadas por la pandemia por el COVID-19, con los resultados se pretende concientizar de la necesidad de dar atención al aspecto psicológico de los docentes ante la carga de actividades excesiva que tienen actualmente por la necesidad de trabajar en una modalidad no presencial.
\end{abstract}

Estrés laboral, Docentes universitarios, Síndrome de Burnout

\begin{abstract}
The article presents an investigation that was carried out with the teaching staff of the Academic Unit of the North of the State of Nayarit (UAEN) belonging to the Autonomous University of Nayarit (UAN), with the aim of carrying out a comparative analysis on the incidence of Burnout syndrome in the years 2018 to 2020. The information collection instrument used was the Maslach Burnout Inventory questionnaire, which measures the following aspects: emotional exhaustion, depersonalization and personal fulfillment. As part of the results, a $10 \%$ increase in the incidence of Burnout Syndrome in teaching staff was documented, which is attributed to the working conditions caused by the COVID-19 pandemic, with the results it is intended to raise awareness of the The need to pay attention to the psychological aspect of teachers given the excessive load of activities that they currently have due to the need to work in a non-face-to-face mode.
\end{abstract}

Work stress, University teachers, Burnout syndrome

Citación: PARRA-GARCÍA, Rosa Ruth, CHÁVEZ-SÁNCHEZ, Haydee del Carmen y ZEA-VERDÍN, Aldo Asunción. El síndrome de burnout en el personal docente de la Unidad Académica del Norte del Estado de Nayarit. Análisis comparativo 2018-2020. Revista de Educación Superior. 2020. 4-12:31-38.

\footnotetext{
*Correspondencia al Autor (Correo Electrónico: ruthparra@uan.edu.mx)

$\dagger$ Investigador contribuyendo como primer autor.
} 


\section{Introducción}

La pandemia del COVID-19 originada en China en diciembre de 2019, ha modificado la forma de vida de toda la sociedad, este virus ha provocado incertidumbre y miedo en la mayoría de la población por los miles de muertos que ha causado. Actualmente, por este motivo, todo el mundo está viviendo momentos difíciles, se han suspendido actividades laborales, económicas, comerciales, recreativas, educativas, entre otras. En este sentido, las instituciones educativas han tenido que readaptar las actividades académicas llevándolas de lo presencial a la virtualidad o clases en línea, lo que no ha sido una tarea fácil, para los estudiantes y sobre todo para los docentes.

Los docentes para cumplir con su práctica educativa, además de realizar sus actividades normales han tenido que habituarse al uso de herramientas tecnológicas, y plataformas que probablemente no utilizaban o no conocían, existen en algunos además de estas inquietudes, presiones emocionales por el confinamiento, en otros una gran preocupación por su salud y la de su familia; lo que puede provocar estrés o síndrome de burnout.

El término estrés laboral de acuerdo con la Organización Mundial de la Salud, es la reacción que puede tener el individuo ante exigencias y presiones laborales que no se ajustan a sus conocimientos y capacidades, y que ponen a prueba su capacidad para afrontar la situación (Leka, Griffiths, \& Cox, 2004).

El síndrome de burnout o síndrome del quemado es un término que se define como "conjunto de síntomas médico-biológicos y psicosociales inespecíficos que se desarrollan en la actividad laboral, como resultado de una demanda excesiva de energía" (Freuedenberger 1974, citado en Ponce, Bulnes \& otros, 2005, p.87). También lo describen como una "pérdida progresiva de idealismo, energía y propósito" (Edelwich y Brodsky 1980, citado por Forbes, R., 2011).
En el año 2018 se realizó una investigación sobre la existencia de este síndrome en el Personal Docente de la Unidad Académica del Norte del Estado de Nayarit donde se reflejó que el $16 \%$ del personal docente de la institución padecía esta enfermedad; sin embargo, con las condiciones actuales por motivo de la pandemia provocada por el COVID-19, se considera pertinente investigar si ese porcentaje se ha modificado (Chávez, Parra, Chávez, \& Hernández, 2018).

El objetivo de la investigación es realizar un análisis comparativo de las manifestaciones del Síndrome de burnout en el personal docente de la UAEN.

\section{Objetivos específicos:}

Identificar los aspectos teóricos del Síndrome de burnout.

- Medir los aspectos de cansancio emocional, despersonalización y realización personal en los docentes de la UAEN.

Diagnosticar el porcentaje de docentes que presentan el síndrome de burnout.

Realizar una comparación de los
resultados.

\section{Síndrome de Burnout}

Maslach y Jackson (1981, 1982) definen el Burnout como "una manifestación comportamental del estrés laboral, y lo entienden como un síndrome tridimensional caracterizado por cansancio emocional, despersonalización en el trato con clientes y usuarios, y dificultad para el logro/realización personal " (Martínez, 2010). Pines y Krafy (1978) lo ven desde una experiencia general de agotamiento físico, emocional y actitudinal. Por su parte Brill (1984) considera que es un estado disfuncional y disfórico relacionado con el trabajo, en una persona que no padece otra alteración y que anteriormente ha desempeñado bien su trabajo y que posteriormente ya no puede conseguirlo. Por otro lado, Gil-Monte y Peiro (2000) consideran al síndrome de burnout como un proceso en el que intervienen componentes cognitivo-aptitudinales, emocionales y actitudinales. 
Por su parte Forbes (2011) menciona que el síndrome de burnout, también conocido como síndrome de desgaste ocupacional o profesional, es un padecimiento que se produce como respuesta a presiones prolongadas que una persona sufre ante factores estresantes emocionales e interpersonales relacionados con el trabajo. Miras (2014), en este sentido, dice que el "Síndrome de Burnout o de agotamiento profesional, significa sentirse quemado, agotado, sobrecargado, exhausto".

En el ámbito de la educación encontramos que éste sindrome es una condición de estrés prolongado e insidioso que se deriva de condiciones del individuo y del contexto organizacional de la institución universitaria (Hederich \& Caballero, 2016).

En cuanto a la posición de la Organización Mundial de la Salud (OMS) hasta antes del 2019, no encontrábamos este padecimiento en su clasificación; fue en la $72^{\mathrm{a}}$ Asamblea Mundial de la Salud celebrada en mayo de 2019; donde se incluyó por primera vez este padecimiento en la Clasificación Internacional y Estadística de Enfermedades y Problemas relacionados son la Salud CIE-11 (Organizacion Mundial de la Salud, 2020), se encuentra con el número QD85 con el nombre de Síndrome de desgaste ocupacional y considerado un problema asociado con el empleo o desempleo. y lo describe como: "resultado del estrés crónico en el lugar de trabajo que no se ha manejado con éxito".

\section{La caracterizan por tres dimensiones:}

- Sentimientos de falta de energía o agotamiento.

- Aumento de la distancia mental con respecto al trabajo, o sentimientos negativos o cínicos con respecto al trabajo.

Eficacia profesional reducida.
De acuerdo con esta clasificación se refiere específicamente a los fenómenos en el contexto laboral y no debe aplicarse para describir experiencias en otras áreas de la vida. Puede tener diferentes formas de manifestación; Gutiérrez, G., Celis, M \& otros (2008) mencionan que sus síntomas son: asilamiento, negación, miedo o temor, depresión, ira, ansiedad, poco interés en las actividades que realizan e irritabilidad.

Forbes (2011) antes mencionado, describe que a nivel psicosocial, genera deterioro en las relaciones interpersonales, ansiedad, depresión, enojo, entre otros; físicamente puede manifestarse presentando insomnio, enfermedades cardiovasculares, pérdida de apetito y peso; problemas gastrointestinales e incluso daños cerebrales; y en el entorno laboral puede afectar la productividad o calidad en el trabajo, la relación con sus compañeros, actitud negativa hacia personas a las que se ofrecen servicios, entre otros.

Por su parte Marrau (2004) dice que la forma de manifestarse es mediante los siguientes síntomas:

Psicosomáticos: alteraciones cardio respiratorias, jaquecas, fatiga crónica, trastornos del sueño, úlceras y desórdenes gástricos, tensión muscular, mareos y vértigos, etcétera.

De conducta: predominio de conductas adictivas y evitativas consumo aumentado de café, alcohol, ausentismo laboral, bajo rendimiento personal, distanciamiento afectivo de enfermos y compañeros y frecuentes conflictos interpersonales en el ámbito de trabajo y de la propia familia.

Emocionales: irritabilidad, incapacidad de concentración distanciamiento afectivo.

Laborales: deterioro del rendimiento, acciones hostiles, conflictos, accidentes, ausentismo, rotación no deseada, abandono. 
Mentales: sentimientos de vacío, agotamiento, fracaso, impotencia, baja autoestima y pobre realización personal. Es frecuente apreciar nerviosismo, inquietud, dificultad para concentrarse y una tolerancia a la frustración con comportamientos paranoides y/o agresivos hacia los pacientes, compañeros y la propia familia.

Maslach (2017), manifiesta que el elemento clave del síndrome es el agotamiento emocional y menciona que los individuos presentan sentimientos de estar emocionalmente agotados y sobrepasados respecto a sus emociones.

Analizando las diferentes opiniones de los autores y considerando que el estrés o síndrome de burnout tiene que ver con aspectos emocionales que pueden afectar el ámbito laboral, consideramos importante identificar si en la unidad académica se ha modificado el porcentaje detectado del síndrome, tomando en cuenta las condiciones actuales de trabajo originadas por la pandemia.

\section{Metodología}

La investigación se llevó a cabo con el personal docente que tiene su adscripción en la UAEN en las licenciaturas en Administración, Contaduría, Mercadotecnia, Ciencias de la Educación y Derecho y se atiende una población estudiantil de aproximadamente 1000 estudiantes, distribuidos en 3 turnos: matutino, vespertino y semiescolarizado.

Se utilizó nuevamente el Cuestionario de Maslach de 1986, que cuenta con 22 ítems en forma de afirmaciones respecto a los sentimientos y actitudes del profesional en su trabajo, el cual fue adaptado para aplicarlo en los docentes y cuyo objetivo es medir el desgaste profesional. Pretende medir la frecuencia y la intensidad con la que se sufre el Burnout. Este instrumento considera 3 aspectos: agotamiento emocional, despersonalización y realización personal.

Se valoran en una escala de frecuencia de siete grados de la siguiente manera:

\begin{tabular}{|r|l|}
\hline \multicolumn{1}{|r|}{ Rango } & \multicolumn{1}{c|}{ Medida de la escala } \\
\hline 0 & Nunca \\
\hline 1 & Pocas Veces al Año o Menos \\
\hline 2 & Una vez al mes o menos \\
\hline 3 & Unas pocas veces al mes \\
\hline 4 & Una vez a la semana \\
\hline 5 & Unas pocas veces a la semana \\
\hline 6 & Todos los días \\
\hline
\end{tabular}

Tabla 1 Escala de Rangos

Para determinar las puntuaciones de cada aspecto se realiza la sumatoria de diferentes ítems de acuerdo con lo siguiente:

\begin{tabular}{|l|r|r|r|}
\hline Categorías & \multicolumn{1}{c|}{$\begin{array}{c}\text { Cansancio } \\
\text { Emocional }\end{array}$} & Despersonalización & $\begin{array}{c}\text { Realización } \\
\text { Personal }\end{array}$ \\
\hline Ítems & $\begin{array}{r}1,2,3,6,8, \\
13,14,16 \text { y } \\
20\end{array}$ & $5,10,11,15$ y 22 & $\begin{array}{r}4,7,9,12,17, \\
18,19 \text { y 21 }\end{array}$ \\
\hline $\begin{array}{l}\text { Indicios de } \\
\text { burnout }\end{array}$ & Más de 26 & Más de 9 & Menos de 34 \\
\hline
\end{tabular}

Tabla 2 Aspectos para evaluar

Los aspectos se miden con los valores de referencia que se presentan a continuación:

\begin{tabular}{|l|r|r|r|}
\hline \multicolumn{1}{|c}{ Aspecto } & \multicolumn{3}{c|}{ Puntuación } \\
\hline $\begin{array}{l}\text { Cansancio } \\
\text { Emocional }\end{array}$ & $0-18$ & $19-26$ & \multicolumn{1}{c|}{ Alto } \\
\hline Despersonalización & $0-5$ & $6-9$ & $10-30$ \\
\hline $\begin{array}{l}\text { Realización } \\
\text { Personal }\end{array}$ & $0-33$ & $34-39$ & $40-56$ \\
\hline
\end{tabular}

Tabla 3 Valores de Referencia

\section{Resultados y conclusiones}

La UAEN cuenta con 26 docentes, que es con quienes se realizó la investigación, de ellos el $50 \%$ son profesores de tiempo completo, $16 \%$ tienen base y el 34\% son docentes de contrato por hora semana mes; las edades oscilan entre 28 y 66 años; el $62 \%$ son mujeres y el $38 \%$ hombres; el $77 \%$ son casados, el $19 \%$ solteros y el $4 \%$ vive con su pareja; respecto al grado de escolaridad cuentan con doctorado el $11 \%$, un $58 \%$ tiene el grado de maestría y el $31 \%$ restante licenciatura.

También es importante conocer si los docentes además del trabajo en la Unidad Académica desempeñan otro tipo de actividad, y en este sentido el $69 \%$ se dedican por completo al trabajo de la Universidad y $31 \%$ realizan una actividad complementaria. En la actualidad el $46 \%$ está realizando estudios de maestría o doctorado y el resto no estudian en este momento. 


\section{Cansancio emocional}

El cansancio emocional valora la vivencia de estar exhausto emocionalmente por el trabajo; considerando los ítems que mides este aspecto se obtuvieron los siguientes resultados: se determinó que el 50\% manifiesta tener un nivel bajo, $31 \%$ medio y solo un $19 \%$ alto.

\section{Cansancio Emocional}

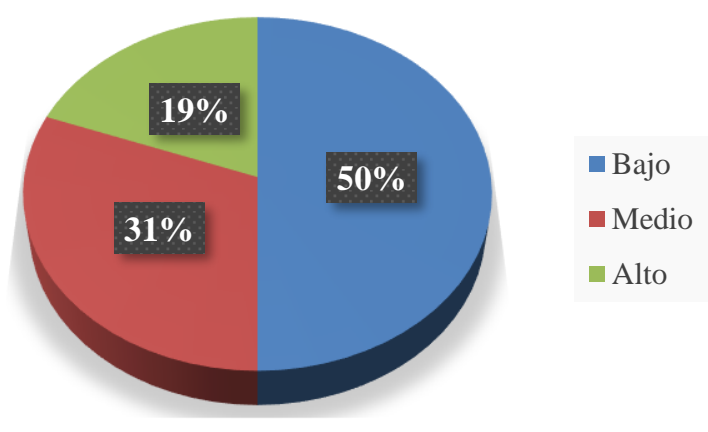

\section{Gráfico 1 Cansancio emocional}

En referencia a los indicios de burnout un $81 \%$ no presenta indicios y el $19 \%$ si manifiesta algo del síndrome.

Indicios de Burnout: Cansancio Emocional

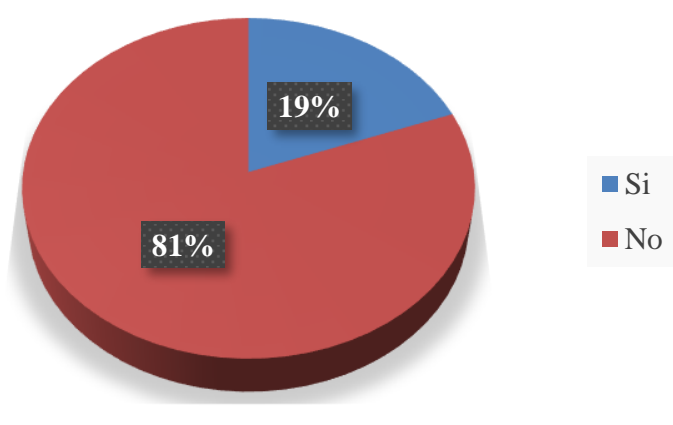

Gráfico 2 Indicios de Burnout en Cansancio emocional

\section{Despersonalización}

Este aspecto valora el grado en que cada uno reconoce actitudes de frialdad $y$ distanciamiento, aquí encontramos que el $69 \%$ presenta un nivel bajo, el $12 \%$ nivel medio y el $19 \%$ restante, un nivel alto.

\section{Despersonalización}

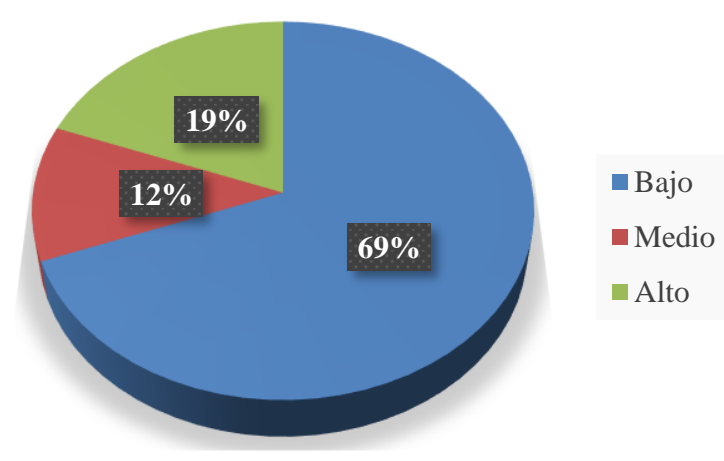

\section{Gráfico 3 Despersonalización}

Tomando en cuenta los valores para detectar indicios del síndrome, vemos que se manifiesta en un $19 \%$ del personal docente y el resto no da señales de padecerlo.

\section{Indicios de Burnout: \\ Despersonalización}

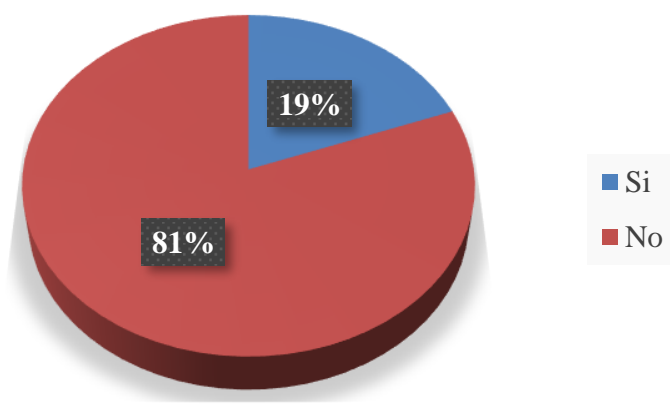

Gráfico 4 Indicios de Burnout: Despersonalización

\section{Realización Personal}

Este aspecto evalúa los sentimientos de auto eficiencia y realización personal en el trabajo y obtuvimos que un $61 \%$ tiene un nivel alto, el $17 \%$ medio y el $22 \%$ bajo.

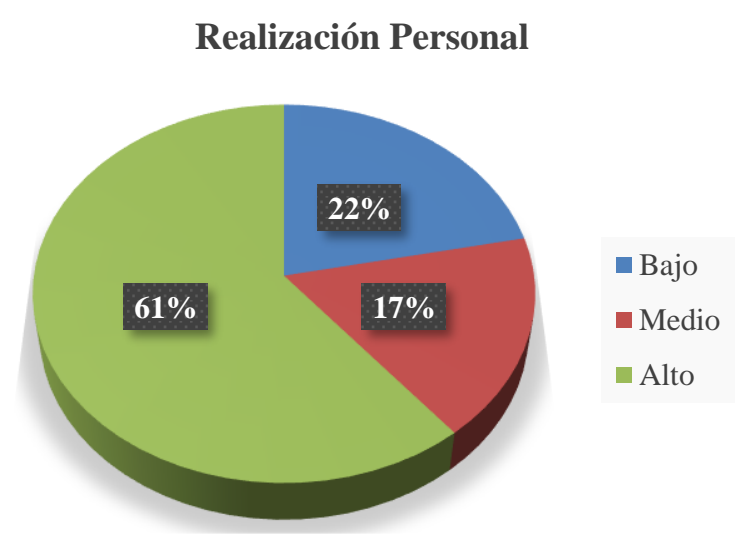

Gráfico 5 Realización Personal

PARRA-GARCÍA, Rosa Ruth, CHÁVEZ-SÁNCHEZ, Haydee del Carmen y ZEA-VERDÍN, Aldo Asunción. El síndrome de burnout en el personal docente de la Unidad Académica del Norte del Estado de Nayarit. Análisis comparativo 2018-2020. Revista de Educación Superior. 2020 
En cuanto a indicios de Burnout el 39\% si lo presentan y el $61 \%$ no tiene señales que nos indiquen que tienen síndrome de burnout.

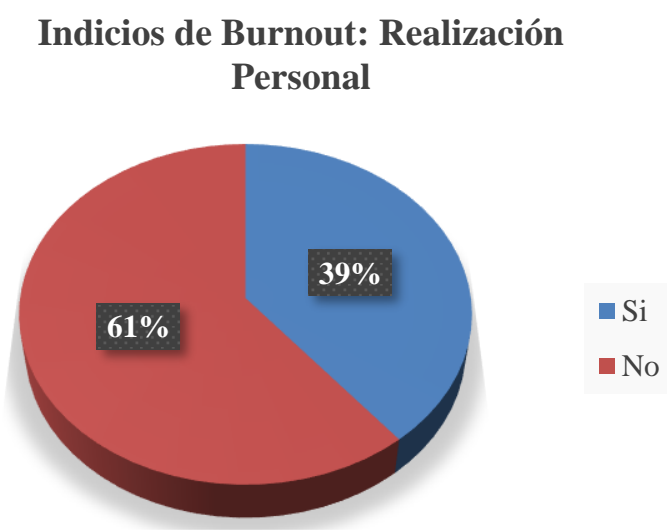

Gráfico 6 Indicios de Burnout: realización personal

\section{Síndrome de Burnout}

Para identificar síndrome de burnout se deben encontrar niveles altos en los dos primeros aspectos y nivel bajo en el tercero; como resultado del análisis de los tres aspectos obtenemos que el $26 \%$ del personal docente presenta Síndrome de Burnout, y el 74\% no.

\section{Síndrome de Burnout}

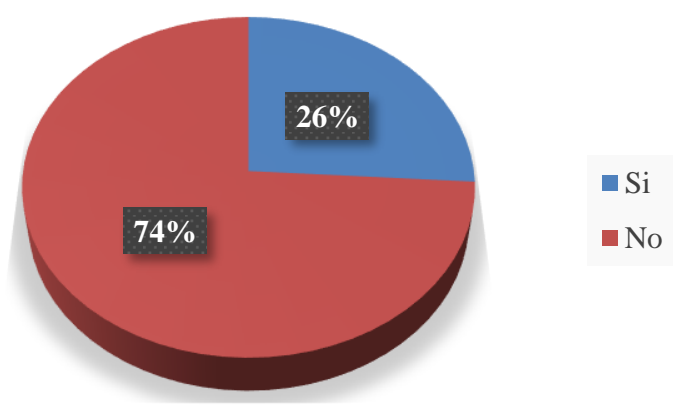

Gráfico 7 Síndrome de Burnout

\section{Análisis Comparativo}

Después de obtener los resultados de la aplicación en la actualidad y en el marco de la pandemia COVID-19 realizamos un análisis comparando cada uno de los aspectos y estos son los resultados. En el aspecto de cansancio emocional se mide el agotamiento que tiene el personal en el trabajo y vemos un aumento de $7 \%$ en el nivel alto, una variación del $12 \%$ al $31 \%$ en el nivel medio y una disminución del $76 \%$ al $50 \%$ en el nivel bajo.

\section{Cansancio Emocional}

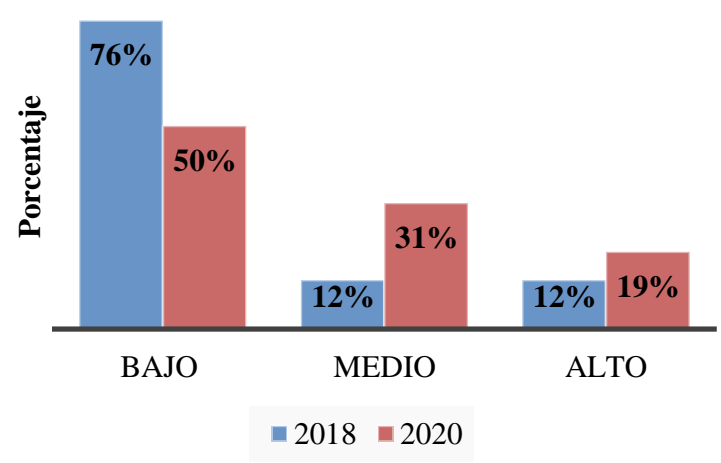

Gráfico 8 Análisis Comparativo: cansancio Emocional

\section{Indicios de Burnout: Cansancio Emocional}

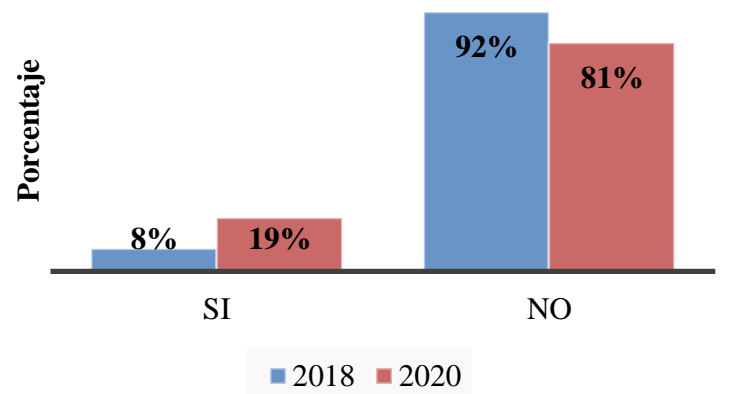

Gráfico 9 Análisis Comparativo: Indicios de Burnout en Cansancio Emocional

Se encuentra un aumento en quienes si presentan indicios de Burnout, el cual sufre un incremento del $11 \%$.

\section{Despersonalización}

En el aspecto que valora el grado en que se reconocen actitudes de distanciamiento y frialdad, nos encontramos con un incremento en el nivel alto del $7 \%$ y un $12 \%$ en nivel medio, respecto a los resultados del estudio previo.

Despersonalización

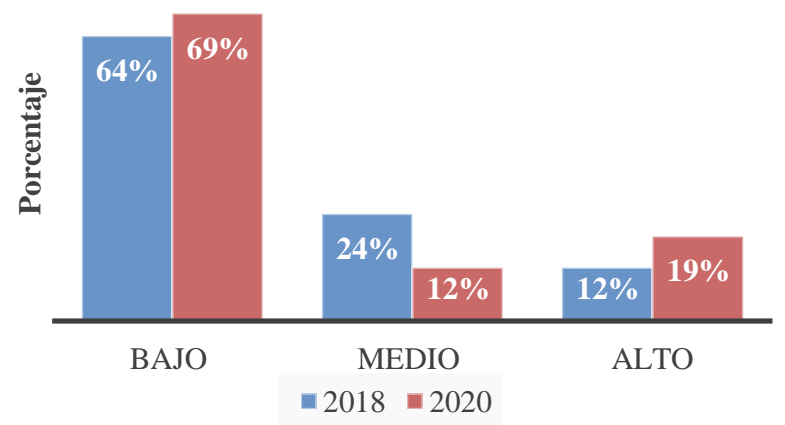

Gráfico 10 Análisis Comparativo: despersonalización

PARRA-GARCÍA, Rosa Ruth, CHÁVEZ-SÁNCHEZ, Haydee del Carmen y ZEA-VERDÍN, Aldo Asunción. El síndrome de burnout en el personal docente de la Unidad Académica del Norte del Estado de Nayarit. Análisis comparativo 2018-2020. Revista de Educación Superior. 2020 
En el caso de despersonalización observamos un aumento del $7 \%$ en quienes, si presentan indicios de burnout, lo cual podemos ver en la siguiente gráfica:

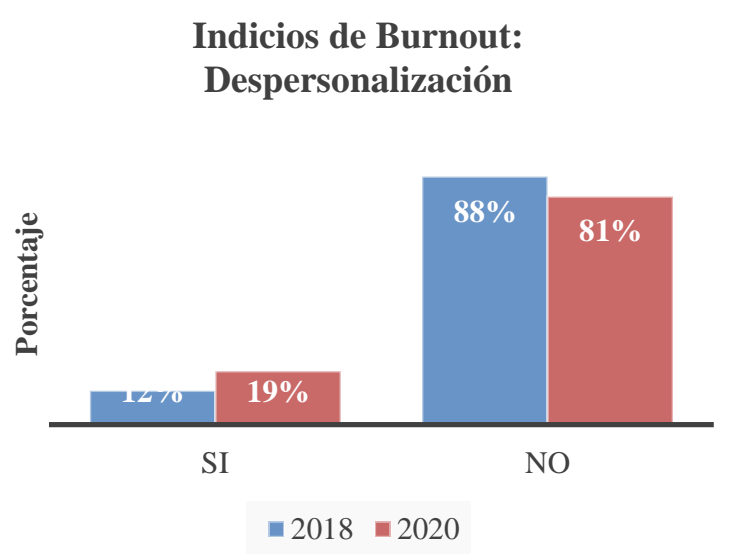

Gráfico 11 Análisis Comparativo: indicios de Burnout en Despersonalización

\section{Realización Personal}

Al analizar la realización personal que evalúa sentimientos de la realización personal identificamos un aumento en el nivel alto de un $21 \%$. Sin embargo, este aspecto es positivo, quiere decir que los docentes se encuentran bien en cuanto a su realización personal en el trabajo.

\section{Realización Personal}

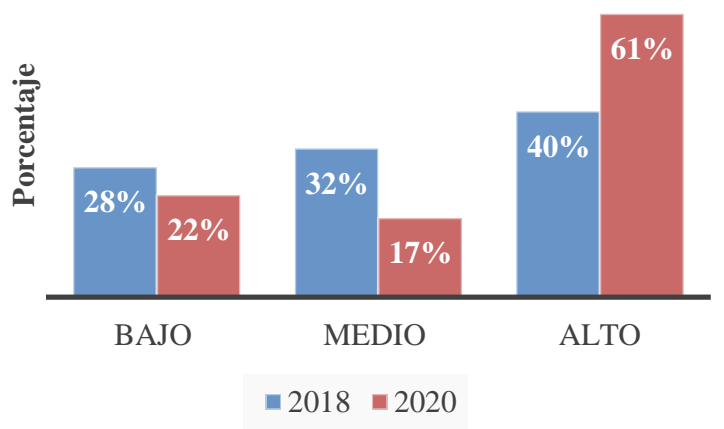

Gráfico 12 Análisis Comparativo: realización personal

Al momento de identificar indicios de burnout, observamos que aun cuando se sienten bien respecto a la realización personal, existe un aumento de un $7 \%$ de quienes si lo manifiestan.

\section{Indicios de Burnout: Realización Personal}

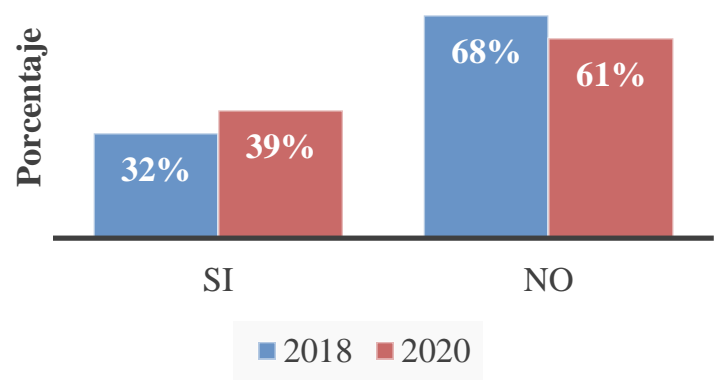

Gráfico 13 Análisis Comparativo: indicios de Burnout en Realización Personal

\section{Síndrome de Burnout}

El resultado respecto a la existencia del Síndrome de Burnout, existe un aumento de un $10 \%$ en los docentes que si tienen algún grado en comparación que la investigación anterior.

\section{Síndrome de Burnout}

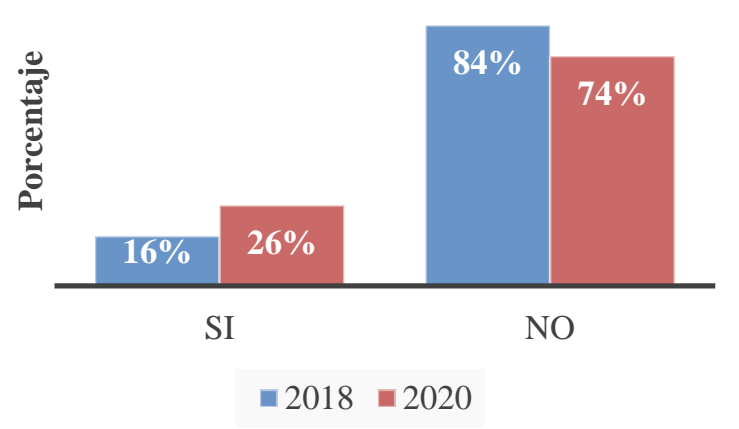

Gráfica 14 Análisis Comparativo: síndrome de Burnout

Considerando todo lo anterior podemos decir que los objetivos de la investigación fueron cumplidos, ya que se analizaron los aspectos teóricos del síndrome burnout, se midieron los aspectos referentes al cansancio emocional, despersonalización y realización personal en el marco de la pandemia; y al comparar los resultados de la investigación con la anterior, observamos que había un $16 \%$ que presentaban el Síndrome de Burnout y en la actualidad el $26 \%$ lo manifiestan, teniendo un aumento de un $10 \%$, por lo cual concluimos que el confinamiento por la pandemia COVID-19, el uso de tecnologías con las que no se estaba familiarizado, la preocupación por la salud y la nueva normalidad están afectando el desempeño de los docentes universitarios de la UAEN. 
Se deben considerar los aspectos documentados en esta investigación, para la asignación de las actividades y buscar estrategias para tratar de reducir o contener los casos de Síndrome de Burnout en este centro de trabajo.

\section{Referencias}

Chávez, H., Parra, R. R., Chávez, G., \& Hernández, J. (2018). Síndrome de Burnout en el Personal Docente de la Unidad Académica del Norte del Estado de Nayarit. Educateconciencia, 9(20), 6-17.

Forbes, R. (2011). El Síndrome de Burnout: síntoms, causas y medidas de atención de la empresa. Éxito Empresarial(160), 1-4.

Hederich, M. C., \& Caballero, D. C. (2016). Validación del cuestionario Maslach Burnout Iventory-Sutdent Survey (MBI-SS) en contecto académic colombiano. Revista CES Psicología, 9(1), 1-15.

Leka, S., Griffiths, A., \& Cox, T. (2004). La Organización del Trabajo y el Estres. Reino Unido: Organización Mundial de la Salud.

Martínez, P. A. (2010). El Síndrome de Burnout. Evolución Conceptual y Estado actual de la cuestion. VivatAcademia(112), 42-80. Obtenido de Martínez Pérez, Anabella (2010). EL SÍNDROME DE BURNOUT. EVOLUCIÓN CONCEPTUAL Y ESTADO ACTUAL DE LA CUESTIÓN. Vivat Academia, (112),42-80.[fecha de https://www.redalyc.org/articulo.oa?id=525752 962004

Mirás, C. S. (2014). Evaluación del Síndrome de Burnout en un Centro de Salud. Revista de Enfermería CyL, 65-73.

Olivares, F. V. (9 de Spetiembre de 2020). Scielo. Obtenido de https://scielo.conicyt.cl/scielo.php?script=sci_ar ttext\&pid=S0718-

24492017000100059\&lng=en\&nrm=iso\&tlng= en\#aff1

Organizacion Mundial de la Salud. (1 de 10 de 2020). Organización Panamericana de la Salud. Obtenido https://icd.who.int/browse11/1-m/es
Ponce, C. B. (2005). "El síndrome del "Quemado" por estres laboral asistencial en grupos de docentes universitarios. Revista de Investigación en Psicología, 8(2), 87-112. 